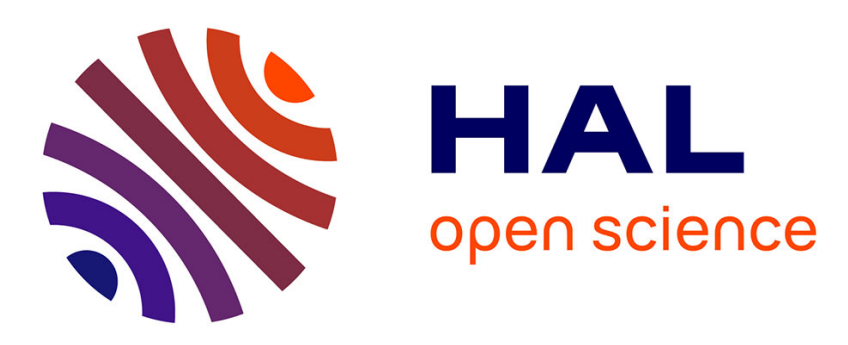

\title{
Statistical Characterization of the Interference on the Return Link of a Broadband Satellite System
}

\author{
Dimitri Serrano-Velarde, Emmanuel Lance, Hector Fenech, Georges \\ Rodriguez-Guisantes
}

\section{- To cite this version:}

Dimitri Serrano-Velarde, Emmanuel Lance, Hector Fenech, Georges Rodriguez-Guisantes. Statistical Characterization of the Interference on the Return Link of a Broadband Satellite System. ESTEL Conference (IEEE-AESS), Oct 2012, Rome, Italy. hal-00732471

\section{HAL Id: hal-00732471 \\ https://hal.science/hal-00732471}

Submitted on 14 Sep 2012

HAL is a multi-disciplinary open access archive for the deposit and dissemination of scientific research documents, whether they are published or not. The documents may come from teaching and research institutions in France or abroad, or from public or private research centers.
L'archive ouverte pluridisciplinaire HAL, est destinée au dépôt et à la diffusion de documents scientifiques de niveau recherche, publiés ou non, émanant des établissements d'enseignement et de recherche français ou étrangers, des laboratoires publics ou privés. 


\section{Statistical Characterization of the Interference on the Return Link of a Broadband Satellite System}

\author{
Dimitri Serrano-Velarde, Emmanuel Lance and Hector Fenech \\ Eutelsat SA \\ Paris, Ile de France, France \\ Email: dserrano@eutelsat.fr, elance@eutelsat.fr and hfenech@eutelsat.fr
}

\author{
Georges Rodriguez-Guisantes \\ Telecom ParisTech \\ Paris, Ile de France, France \\ Email: rodriguez@telecom-paristech.fr
}

\begin{abstract}
This work describes an interference study of the return link (user terminals to gateways) of a high throughput satellite (HTS) for broadband services. The aim is to identify a statistical behavior of the inter-spot interferences due to frequency re-use so as to estimate the carrier over interference ratio $(\mathrm{C} / \mathrm{I})$ on the uplink segment of the return link for a multi frequency time division multiple access (MF-TDMA) technique. The characterization of the interference will be done based on a mathematical model and validated through empirical observations for different transmission scenarios and satellite systems. In this work, we use the $C / I$ performance at satellite antenna level as a benchmark parameter, in order to find the most faithful statistical law for the generated interferences. The satellite architecture itself uses a multi-beam coverage implementing frequency re-use and spatial separation.
\end{abstract}

\section{INTRODUCTION}

Multi-beam satellite coverages are used more and more widely by satellite operators like Eutelsat with KaSat, or Viasat with ViaSat1 in order to gain more flexibility and capacity by reusing certain bandwidths several times at different locations. Frequency re-use increases the overall satellite capacity even when only small bandwidths are available. MF-TDMA access techniques are in addition a useful approach to divide in time and frequency the accesses to the spectral resources from the various user terminals for the uplink segment of the return link i.e. user terminals to the satellite. The spectral resources are fully used as each user terminal access the complete spectral resources at its disposal for a certain period of time in order to transmit its data to the corresponding gateways.

In addition, this coverage and frequency flexibility can have multiple benefits:

- improved link budget performances through more directive beams;

- traffic benefits with the implementation of "hot spots" (i.e. spots with high traffic loads);

- commercial benefits with a reduced cost per Gbps (i.e. the satellite's "financial" efficiency);

- addressing new/bigger markets.

There are however several issues related to this kind of coverage. The interference generated between spots using the same frequency bands is a major drawback. To name a few, the spot pattern or the satellite reflectors are crucial design elements that can either compensate losses or if poorly chosen, increase the interference. Indeed, the spot pattern defines the spatial proximity of all the spots using the same bandwidth and frequencies and the satellite antenna design conditions the directivity and the side-lobe effects of each beam. Analyzing and characterizing the interference through a statistical law, enables the possibility to quickly calculate their intensity without going through long calculations. With this statistical model, it is possible to uncorrelate the interference generated by different user transmission scenarios (different active user positions) and integrate all the possibilities without having to go into heavy and time consuming simulations.

In Section II, we present the problematics of the interference study in terms of C/I. In Section III, we introduce the key system models and explain the hypothesis that had been chosen for the calculations. In Section IV, we analyze the interference and conclude on possible channel capacity models. Section V, gives a perspective of further possibilities and concludes the work.

\section{PRoblem DESCRIPTION}

Satellites have long been used for services related to broadcasting like TV or radio but the newer generation aims at providing broadband applications as a natural extension of interactive services. This move is especially encouraged with the use of new frequency bands such as the Ka band providing more directive antenna patterns, new usable bandwidths and smaller user terminal antennas.

For this kind of applications, the satellite architecture mainly used is a multi-beam coverage composed of a given number of beams and a corresponding cell structure, which once put together, generate the whole service area.

Through frequency re-use and by separating spatially the beams using the same frequencies, it is possible to re-use a limited bandwidth several times, increasing the overall system capacity. A more complex approach, is to allocate variable frequency bandwidths to every spot maximizing at each instant the spectral resources and stabilizing the service quality but this solution implies a high complexity at satellite level. Moreover a MF-TDMA access technique helps to share even more the resources between all the different active user terminals in time and frequency. Each user terminal will access during a given period of time the whole spectral resources allocated to him.

However, the major drawback of such architectures is the inter-spot interference and the need to properly control 
their level. As opposed to the forward link, the interferences are dynamic. At each instant, the active user terminal constellation varies. It is possible that it can be either a different user at a different location or the same user at the same location or no user at all. The classic method of calculating this kind of interference is through long MonteCarlo simulations, generating each time new possible user terminal constellations over time. This process, based on an ergodic hypothesis of the traffic, is however time consuming and needs heavy computer resources.

Besides, in the early stages of a satellite project, using a quick and relative accurate method to estimate the interference is needed in order to make the right design choices, accelerating the whole satellite design process. To find the appropriate model, an empirical approach has been adopted. Consequently, it is necessary to collect the data, analyze it, select a suitable model and validate it. Furthermore, once the distribution is known, it is possible to find a model for the channel capacity in order to get a better grasp of the specificity of the satellite link [1].

Consequently, the problematic for this paper concerns the the characterization of the inter-spot interference so as to apprehend the interference environment through empirical methods and in the end through a statistical law. This will allow a certain channel capacity model.

\section{Proposed Key Models}

\section{A. Antenna Model}

In our work, one of the antenna design choice is to keep the focal length to diameter ratio (f/a) constant to one. The reflector size, $a$, has to be chosen and also kept fixed for the whole performance assessment of the methodology.

In this work we use a classic antenna model, which can be easily characterized in terms of equivalent isotropically radiated power (EIRP) and gain over noise temperature figure of merit. The adopted reflector model takes into account the illumination taper (the amount of energy reflected by the aperture) and more precisely the edge taper and spillover losses. The antenna trade-off is a very complex process and results from different considerations ranging from the defined service area to the size of the reflector and feed geometry. The consequences of a poor antenna choice conditions the overall satellite performances in terms of C/I. So, including a coherent antenna model into the methodology, translates the effects (benefits or losses) of the number of beams for a given reflector size.

The chosen model for our study is based on the work done by Peter Balling [2] but more antenna models and simulation samples can be found in the references [3] and [4]. Balling's mathematical model propose the antenna gain as a linear combination of Bessel functions and some corrective coefficients that takes into account the spillover and edge taper illumination. The position of the beams and their relative direction towards the reflector are also integrated.

Let us consider a feed $j$ in a cluster and the reflector geometry shown in Figure 1.
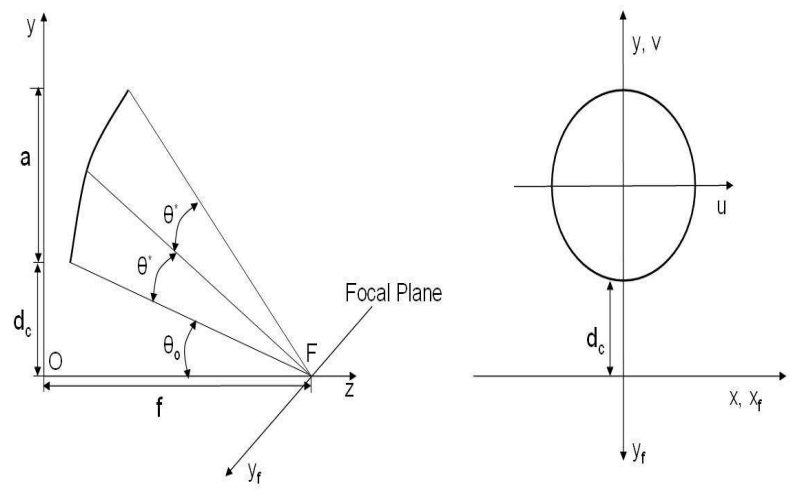

Figure 1. Side and front views of offset reflector with focus at $\mathrm{F}$ and $x_{f} y_{f}$ focal plane

The field amplitude generated by a feed can be expressed as:

$$
\begin{gathered}
F_{j}(\theta, \phi)=\kappa \times a \times\left(c_{1} \times \chi\left(1, \kappa \times a \times x_{j}\right)+\right. \\
\left.c_{2} \times \chi\left(n+1, \kappa \times a \times x_{j}\right)\right)
\end{gathered}
$$

with:

- $a$, reflector diameter,

- $\kappa$, the propagation constant $\frac{2 \pi}{\lambda}$,

- $\theta$ and $\phi$, coordinates in a spherical reference system with the antenna boresight as the zenith direction,

- $x_{j}$, the angular distance between the field direction $(\mathrm{u}, \mathrm{v})$ and the feed, $x_{j}=\sqrt{\left(\left(u-u_{j}\right)^{2}+\left(v-v_{j}\right)^{2}\right)}$,

- $(u, v)$ the coordinate system defined as $u=$ $\sin \theta \cos \phi ; v=\sin \theta \sin \phi$

- $n$, number of feeds,

- $\chi$ is given by:

$$
\chi(n, x)=\left\{\begin{array}{l}
1, x=0, \\
2^{n} n ! \frac{J_{n}(x)}{x^{n}}, \forall x \neq 0 . \\
J_{n}(x)=\frac{1}{2 \pi} \times \int_{-\pi}^{\pi} \exp [-i(n \tau-x \sin \tau)] \mathrm{d} \tau \\
J_{n}(x) \text { is a Bessel function of order } \mathrm{n}
\end{array}\right.
$$

The model is dependent on the number of feeds to accommodate and the coordinates of each one, with regard to the reflector. In addition to the feed cluster geometry, those elements are taken into account in the corrective coefficients $c_{1}$ and $c_{2}$ given by:

$$
\begin{gathered}
c_{1}=\frac{\zeta_{o}}{\sqrt{\frac{1+n+2 \times n \times \zeta_{o}+2 \times n^{2} \times \zeta_{o}^{2}}{1+3 \times n+2 \times n^{2}}}} \times L_{S O} \\
c_{2}=\frac{1-\zeta_{o}}{(1+n) \sqrt{\frac{1+n+2 \times n \times \zeta_{o}+2 \times n^{2} \times \zeta_{o}^{2}}{1+3 \times n+2 \times n^{2}}}} \times L_{S O}
\end{gathered}
$$

with:

- $\zeta_{o}$, average aperture edge illumination i.e reflected power

- $L_{S O}$, element-beam spillover loss

Finally, the antenna directivity $D_{i r}$ in $\mathrm{dB}$ is:

$$
D_{i r}=10 . \log _{10}\left(\left|F_{j}(\theta, \phi)\right|^{2}\right)
$$


By integrating a certain conversion factor, one can obtain the gain over noise figure of merit:

$$
\frac{G}{T}=D_{i r}-C_{\text {factor }}
$$

with:

$$
\text { - } C_{\text {factor }}, \text { conversion factor in } \mathrm{dB}
$$

\section{B. Interference Model}

In a multi-beam coverage with frequency re-use, there will be two types of interference: the interference induced by the spots using the same frequency with the same polarization but spatially separated, called co-polar interference $I_{c o}$, and the interference induced by the spots using the same frequency but with a directly opposite polarization, called cross-polar interference $I_{c x}$.

Based on a Monte-Carlo approach, let us consider for a given user constellation, at satellite level, the receiving pattern of a spot $k$, receiving the signal of its respective active user terminal with a power $C_{k}$ (in $\mathrm{W}$ ) at position $x_{s}$ but it also receives the signals from the neighboring active user terminals at position $x_{C o}$ for the co-polar interferers and $x_{C x}$ for the cross-polar interferer, each using the same frequency bands.

The Co-polar contribution $I_{c o}\left(x_{C o}\right)$ can be evaluated as:

$$
I_{c o}\left(x_{C o q}\right)=\sum_{q=1}^{M_{I_{C o}}} P_{c o}\left(q, x_{C o q}\right),
$$

with:

- $q$, refers to the $q^{s t}$ interferer spot,

- $M_{I_{C o}}$, the total number of interferers in co-polarization,

- $x_{C o q}$, position of the user terminal,

- $P_{c o}\left(q, x_{C o q}\right)$, the received power by the satellite for the $q^{s t}$ interferer in co-polarization at position $x_{c}$.

The cross polarization contribution, $I_{c x}\left(x_{C x}\right)$, can be evaluated as:

$$
I_{c x}\left(x_{C x m}\right)=\sum_{p=1}^{M_{I_{C x}}} P_{c x}\left(m, x_{c x m}\right),
$$

with:

- $m$, refers to the $m^{s t}$ interferer spot,

- $x_{C x m}$, position of the user terminal,

- $M_{I_{C x}}$, the total number of interferers in the cross polarization,

- $P_{c x}\left(p, x_{C x m}\right)$, the received power by the satellite for the $m^{s t}$ interferer in cross polarization.

Thus for spot number $k$ :

$$
\begin{gathered}
I_{k}\left(x_{c}\right)=I_{c o}\left(x_{c}\right)+I_{c x}\left(x_{c}\right) \\
\left(\frac{C}{I}\right)_{k}\left(x_{s}\right)=\frac{C_{k}\left(x_{s}\right)}{I_{k}\left(x_{c}\right)}
\end{gathered}
$$

This process needs to be repeated over a large sample of user constellations so as to obtain an average $C / I$ value. Also, note that we consider the interference behavior identical in both polarizations.

\section{RESUlts}

In this Section, we present the results of the interference study given for a fixed service area and antenna system. The satellite provides a service over an elliptical polygon covering mainly France from an orbital position of $0^{\circ} \mathrm{E}$. The antenna system is composed of four identical reflectors of $2.8 \mathrm{~m}$ (state of the art reflector size) and the number of included spots is allowed to vary over the area. Each spot pattern will be divided into a cell structure based on the best G/T performance available. Consequently each cell is composed of a pool of uniformly distributed user points at various geographical positions in the cell. During the interference calculation, a user is randomly chosen in each cell so as to build a given user constellation for one instant. Note that every user terminal is considered identical in terms of antenna size and possible performances. The frequencies used are 19.7 GHz and $20.2 \mathrm{GHz}$ in Ka-Band with four colors. The air interface is the DVB-RCS standard.

An example of such a coverage in G/T and active user terminal constellation is shown in Figure 2. Active user terminals are represented with a colored dot, corresponding to the "color" they belong to.

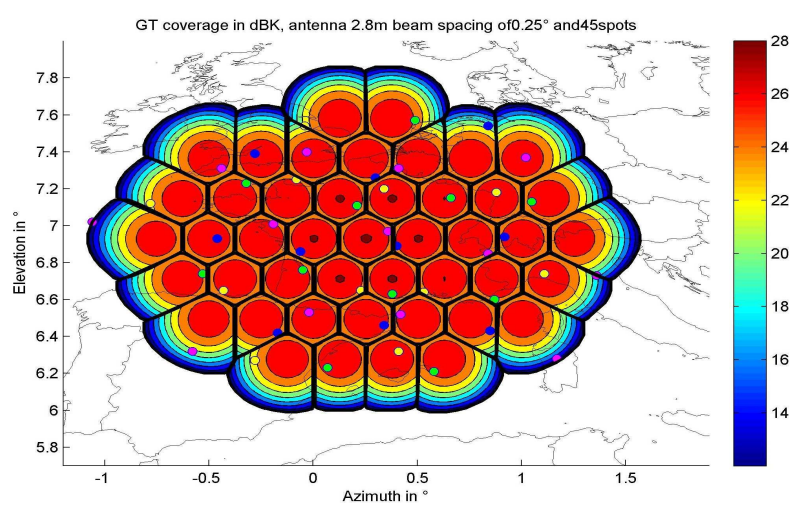

Figure 2. GT service area coverage over France for 45 spots with an active user constellation

For the sake of clarity, the analysis will be based on data provided by three different beam densities: 93 spots, 83 spots, 60 spots and 45 spots (others have been calculated).

To begin with, the interference will be calculated through a Monte Carlo process for every beam in the coverage, with a high number of active users i.e. satellite at full load. Then through a curve fitting process based on maximum likelihood estimators, the distribution of the interference levels in Watt is estimated so as to obtain the mean, $\mu$ and the standard deviation $\sigma$ of the distributions. For this study, only two statistical distributions have been kept, after a pre-selection process where we compared various distributions with the empirical data. The distributions are a Lognormal and Normal distribution. One can see an example of curve fitting in Figure 3. 


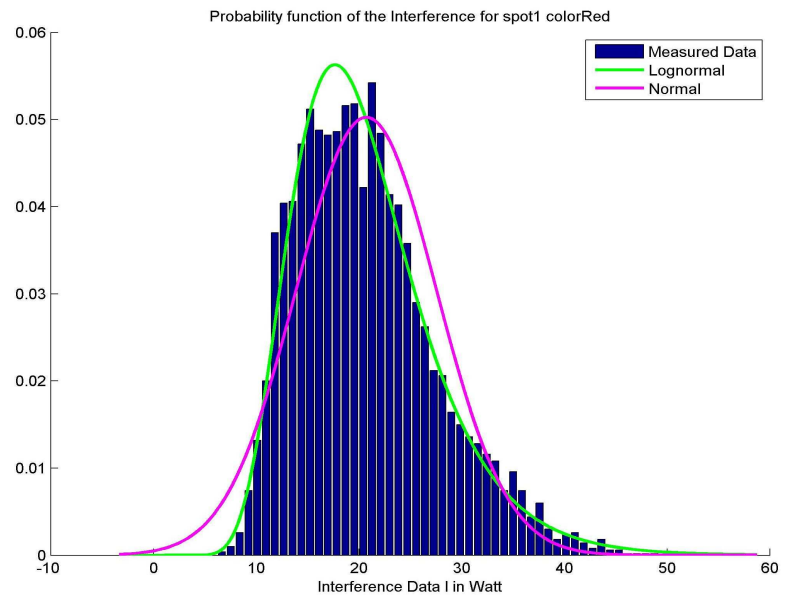

Figure 3. Distribution curve fitting for a studied spot

It is already possible to consider that a Lognormal distribution appears to be the best fit. By using the cumulative distribution functions (CDF) with the obtained $\mu$ and $\sigma$ and calculating the absolute difference, the Lognormal is the best fitting law. The difference between the measured data and the theoretical data being smaller compared to the Normal distribution.

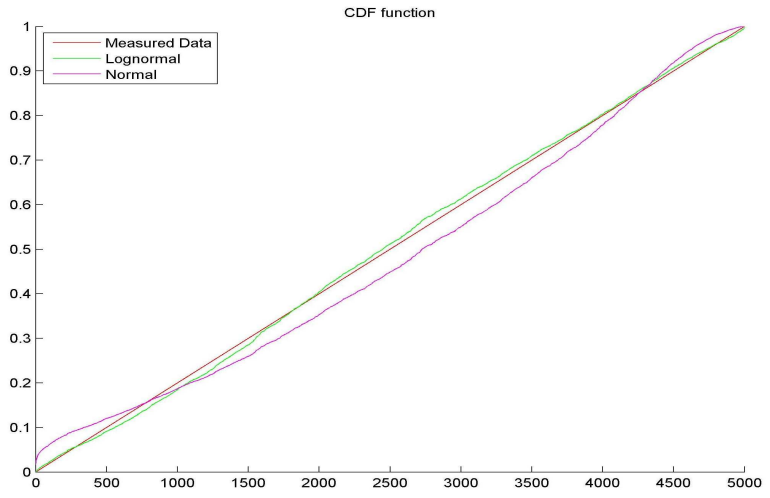

Figure 4. Distribution curve fitting for a studied spot

This comparison process has been performed for all the spots of each multi-beam coverage scenario of 93 spots, 83 spots, 60 spots and 45 spots in order to characterize the two distributions each time in terms of mean and standard deviation. It appears that for all three scenarios $71 \%$ of the cases, the best fit is a Lognormal distribution and $21 \%$ are fitted by a Normal distribution.

This supports a preliminary conclusion: the Lognormal law seems to characterize the most appropriately the interference environment on average.

In a second time, each distribution will be compared to the worst case values in terms of $I, C / I$ and $C /\left(N_{0}+I\right)$. By definition, the worst case in a HTS satellite communication, corresponds to the case where for each active user, the interference is generated by all the highest contributors at the worst positions, same frequency and same time. Comparing those two approaches, will highlight two points:
- the fundamental difference between a standard value and the worst case value (often considered for a satellite specification)

- the impact of choosing a Lognormal or Normal distribution

During this evaluation process, it is possible to characterize the difference in terms of $I, C / I$ and $C /\left(N_{0}+I\right)$ with a Gaussian distribution. Indeed, we assume that the difference is Gaussian [5]. In order to evaluate the uncertainty, we will generate a high amount of samples so as to reach an asymptotic behavior and compare the values to the worst case. The process based on a statistical law is much faster and the results are the average value over the four "colors".

Figure 5 and Figure 7 show the average difference between the values obtained by the distributions and the worst case values in terms of the interference $I$ and the obtained $C / I$. It appears that the estimations for the interference $I$ with the Lognormal and Normal distribution are around $3 \mathrm{~dB}$ to $8 \mathrm{~dB}$ better. However, the difference between the estimated and worst case values varies with the beam density. For lower beam densities, the gain is more pronounced than for higher beam densities. This is mainly due to much more directive radiation diagrams for smaller beams. The smaller the beam is, the smaller the antenna feed becomes. Thanks to smaller feeds, the side lobe levels are lower so that the worst contribution is generally lower compared to the side lobe levels generated by bigger feeds. However with higher beam densities, the number of interference contributions is also higher. For the $C / I$ performance, as the interference $I$ is less stringent on a nominal basis (distributions), one gains around $3 \mathrm{~dB}$ for high beam densities and $6 \mathrm{~dB}$ for lower beam densities. Again the importance of the radiation diagram and of the number of interferers is highlighted. Consequently, considering the worst case, implies very stringent system constraints, blocking out possible benefits on a link budget and related architectural spacecraft benefits.

Last but not least, by considering a $C / N_{0}$ varying between $10 \mathrm{~dB}$ to $18 \mathrm{~dB}$, the final $C /\left(N_{0}+I\right)$ is generally improved. For low $C / N_{0}$ values, the systems are noise limited (especially for low beam densities) and there is only a limited increase of more than $1 \mathrm{~dB}$ in terms of $C /\left(N_{0}+I\right)$. For higher $C / N_{0}$ values, the system is reaching an equilibrium between the thermal noise and the interference, gaining up to $3.5 \mathrm{~dB}$. So using the worst interference case as a satellite design baseline, implies excessive constraints in prevision for a user constellation that is rarely happening. 


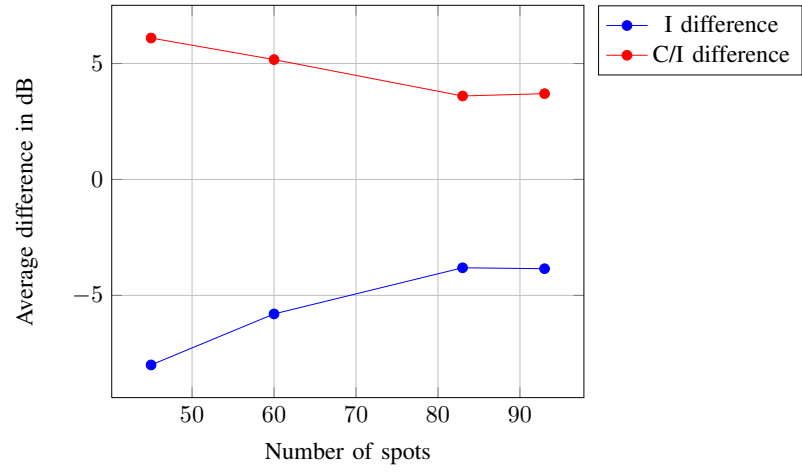

Figure 5. Average difference in terms of I and C/I for the lognormal distribution
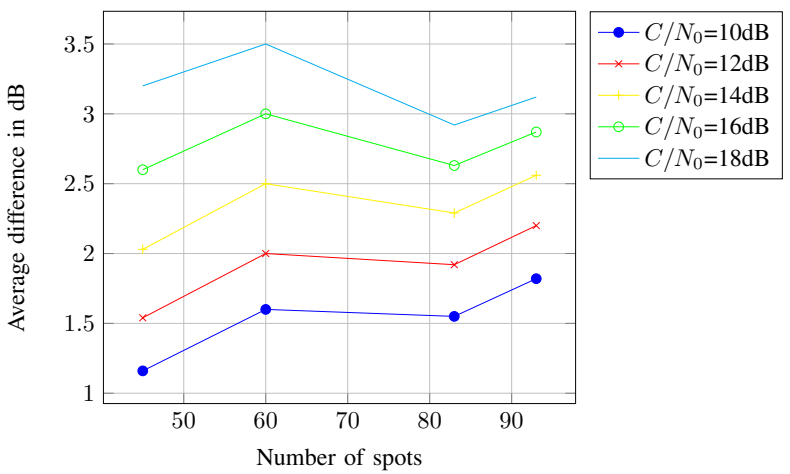

Figure 6. Average difference in terms of $\mathrm{C} /\left(N_{0}+\mathrm{I}\right)$ for the normal distribution

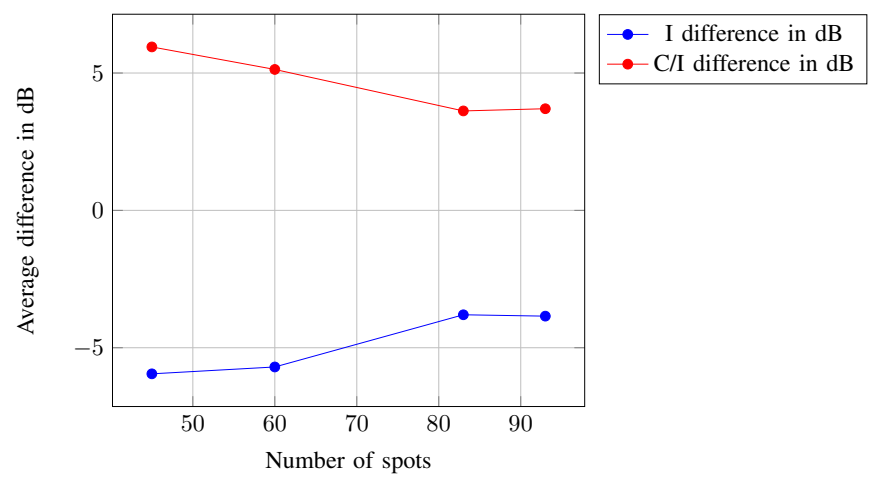

Figure 7. Average difference in terms of I and $\mathrm{C} / \mathrm{I}$ for the normal distribution

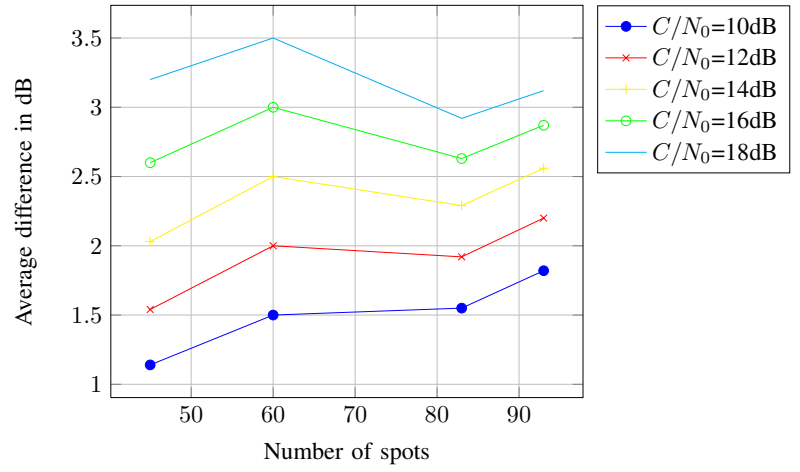

Figure 8. Average difference in terms of $\mathrm{C} /\left(N_{0}+\mathrm{I}\right)$ for the normal distribution

Figure9 and Figure 10 shows the possible gains in terms of spectral efficiency. It appears that the gain in efficiency is less for lower beam densities. Basically, for lower beam densities, each interferer is relatively far from each other, so that even in a worst case scenario, the $\mathrm{C} / \mathrm{I}$ values are acceptable. However, for high beam densities, the difference is more stressed out as the beams are closer. The worst case for this kind of spot pattern is much more stringent (even with more directive beams) and each "won" $\mathrm{dB}$ has a higher impact on the overall performance.

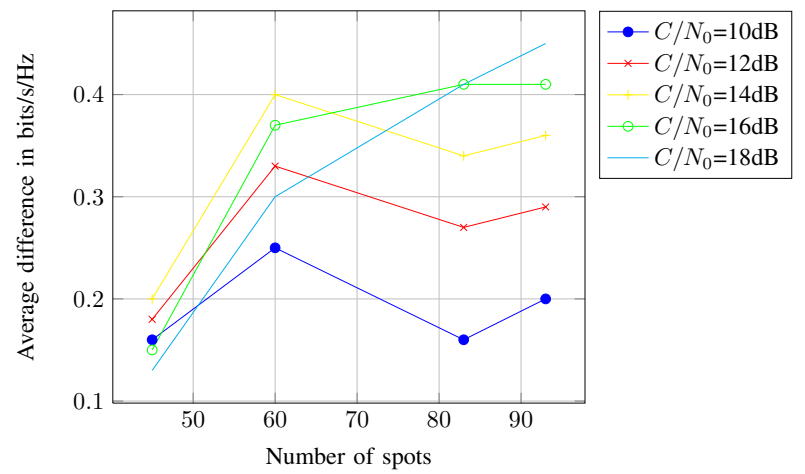

Figure 9. Average difference in terms of spectral efficiency for the lognormal distribution

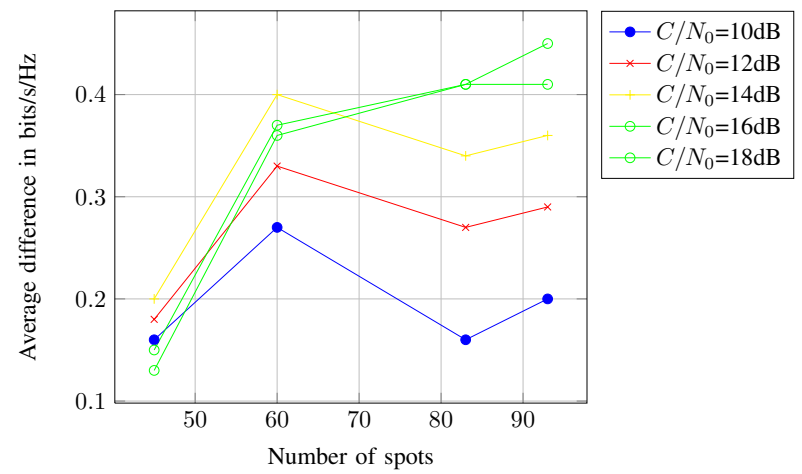

Figure 10. Average difference in terms of spectral efficiency for the normal distribution 
Finally, it appears that choosing either a Lognormal or Normal distribution does not not imply heavy differences even if the Lognormal is the most fitting distribution. Furthermore, by choosing a Normal distribution in order to characterize the interference the channel capacity defined by Shannon is applicable. The channel capacity for the additive white Gaussian noise (AWGN) model can be calculated as [6]:

$$
C=B \times \log \left(1+\frac{S}{N+I}\right)
$$

with:

- $C$, channel capacity in bits per second;

- $B$, bandwidth of channel in $\mathrm{Hz}$;

- $S$, total signal power on bandwidth $B$;

- $N$, thermal noise power on bandwidth $B$;

- $I$, interference on bandwidth $B$.

In addition, to faster processing times, a normal distribution is advised for later use as the mathematical model is well known. So, basing a satellite design or specification on the worst case scenario in terms of interference implies higher system constraints for a rare and specific case. Being able to ease the $\mathrm{C} / \mathrm{I}$ requirement opens more efficient system tradeoffs for lower costs and mass. Using a nominal interference case provides more efficient and balanced systems.

\section{Conclusions}

This work is a first step in the characterization of the interference environment on the return link of a broadband satellite communication. It has highlighted the benefits of characterizing the interference environment by a statistical law. On one side, the interference estimations are faster and on the other side, the interference levels can be decorrelated from any active user constellation. Furthermore, it has been highlighted the benefit of using a nominal design case rather than a worst case. The differences are present, meaning that using the worst case implies more stringent system constraints. Also it is important to note the relationship between the interference and the position of each beam within a coverage. Further work will be done in order to characterize the distributions with regard to the properties of a multi-beam coverage in terms of angular distances and spot pattern.

\section{REFERENCES}

[1] G. K. K. Nikos C. Sagias, George S. Tombras, "New Results for the Shannon Channel Capacity in Generalized Fading Channels," IEEE Communications Letters, vol. 9, 2005.

[2] P. Balling, "Spacecraft Multi-Beam and Contoured-Beam Antennas," AGARD lecture: Microwave Antennas for Avionics, no. 151, p. 24, 1987.

[3] S. N.Makarov, Antenna and EM Modeling with Matlab. John Wiley and Sons, Inc., 2002.

[4] C. A. Balanis, Antenna Theory 3rd Edition, Analysis and Design. John Wiley and Sons, Inc., 2005.

[5] W. Feller, An Introduction to Probability Theory and Its Applications. John Wiley and Sons, Inc., 1971, vol. 2.

[6] R. E. Blahut, Principles and Practice of Information Theory. AddisonWesley Publishing Company, Reading, MA, 1987. 